\title{
LA ADMINISTRACIÓN ELECTRÓNICA EN LOS MUNICIPIOS DE TOluCA Y MetepeC, Estado de MÉXICO
}

\author{
Juan Miguel Morales y Gómez \\ jumimogo@yahoo.com.mx
}

\section{Leticia Contreras Orozco}

lcorozco2003@yahoo.com.mx

\section{Leobardo Ruiz Alanís}

leorualanis@yahoo.com

José JuAn SÁnchez GonzÁlez

cameralista@hotmail.com

Universidad Autónoma del Estado de México

El trabajo busca conceptualizar la administración electrónica, sus elementos y características para analizar los avances de dos municipios del Estado de México. Se argumenta que la administración electrónica permite la optimización en la prestación de los servicios, mejora el acceso a la información, genera economías de escala y reduce el costo unitario de cada operación del gobierno, mejorando la capacidad de respuesta a los ciudadanos. De la comparación de ambos casos, se concluye que han existido avances importantes pero que aun queda mucho por hacer para mejorar, por esta vía, la vinculación entre el ciudadano y la administración pública.

Palabrasclave: administración electrónica, gobierno electrónico, modernización, Tecnologías de la información y comunicación.

\section{ELECTRONIC MANAGEMENT IN THE MUNICIPALITIES OF TOluCA AND METEPEC, STATE OF MEXICO}

This paper attempts to conceptualize the elements and characteristics of electronic management in order to analyze progress in two municipalities of the State of Mexico. We argue that electronic management allows for the optimization of service provision, improves access to information, generates economies of scale and reduces the unit cost of each government operation, improving citizen response capacity. From the comparison between both cases, we conclude that there has been important progress but there is still much that can be done to improve the link between the citizen and public administration.

Keywords: e-administration, e-government, modernization. 


\section{INTRODUCCIÓN}

No se pueden ocultar, o dejar a un lado, los procesos de modernización, ya que están inmersos en cada uno de los espacios que nos rodean, se hacen existentes a través de los medios que utilizamos cotidianamente. Incluso, se puede decir que los procesos de modernización se han venido desarrollando desde hace más de dos siglos construyendo las herramientas que le han permitido consolidarse, en los ámbitos económico, cultural, social y político. En la administración pública la modernización se ha visto como un proceso necesario para promover la efectividad de la acción gubernamental y a su vez ésta conduce a la administración pública a acentuar su transformación. Es decir, se presenta ahora la dinámica de mutua influencia.

En este tránsito, la aparición de las computadoras en la década de 1970 y de internet en la de $1980^{1}$ se ha considerado como herramienta clave para el desarrollo y la competitividad de las instituciones, así como para atender la necesidad permanente de comunicación entre los ciudadanos con la administración pública. El uso de las técnicas de la información y comunicación se ha intensificado de tal forma que está cambiando la imagen que el ciudadano tenía de la administración pública, dando lugar en los países más desarrollados a una especie de competición por innovar en los servicios e información facilitados al público, lo que en Europa se denomina administración (pública) electrónica ya que tiene un alto desarrollo en aspectos administrativos.

El presente artículo contempla una recopilación de diversos conceptos aportados por autores reconocidos sobre modernización administrativa, y sobre Tecnologías de la Información y Comunicación (TIC), se incluyen avances significativos sobre la conceptualización de la administración electrónica, sus elementos y características, tan favorables a la transparencia y al acceso a la información para construir esquemas cada vez más profundos en la interacción entre ciudadanos y gobierno. Esta investigación también describe y analiza los logros que en materia de administración electrónica se han venido desarrollando en el mundo y

1 Se crea primero Arpanet en 1969, después se da la separación de la red del Departamento de Defensa de EEUU, bajo el nombre de Milnet en 1983. La implementación de estos conceptos empezó a finales de los ochenta; en esta década las tecnologías se reconocen como bases de la moderna Internet. En los noventa se introdujo la World Wide Web, que no tardó en hacerse de uso común. 
en México, lo cual permitirá apreciar los logros que se tienen hoy en día. En una tercera parte se presenta el resultado de un análisis empírico de administración electrónica en dos municipios del Estado de México los cuales son: Toluca, capital de la entidad, y Metepec ${ }^{2}$, colindante con esta. Asimismo, se analizan a través de sus páginas electrónicas cuáles son sus fortalezas y debilidades en cuanto a sus logros en Administración electrónica, a fin de realizar una comparación de los dos municipios. Finalmente se presentarán las conclusiones y recomendaciones derivadas del trabajo empírico, así como su relación con los sustentos teóricos.

\section{MODERNIZACIÓN ADMINISTRATIVA UNA NECESIDAD DE LOS GOBIERNOS PRESENTES}

Se puede afirmar que las transformaciones en los diferentes países se han dado acorde a su historia; las decisiones que han tomado los gobiernos en el pasado han sido el impulso del presente, al menos hasta el desarrollo de la globalización.

La globalización, puede ser vista como una diversidad de fenómenos que hacen desaparecer cualquier frontera de los espacios geopolíticos, lo cual genera que los países reorienten las estructuras que habían mantenido hasta hace unos cuantos años. Entre tales fenómenos destacan: el proceso de innovación científica y tecnológica, la competencia por los mercados y la búsqueda de nuevos instrumentos de integración de los sectores y el conjunto regional. Es por esto, entre otras cosas, que las administraciones públicas se han planteado modernizar y actualizar su esquema de relaciones, buscando desarrollar mecanismos orientados a fortalecer la eficiencia y transparencia en aquellas funciones operativas y normativas que les corresponde atender. En este contexto, Pardo define a la modernización administrativa como "la adecuación y ajuste de la gestión estatal para lograr que el flujo de acciones sociales predominantes en una sociedad logre la coherencia y organización necesarias" (Pardo, 1992:19).

Probablemente una de las más completas definiciones de modernización es la que expone Eisenstadt (1972:7), quien señala que la modernización es la creación de

2 Metepec es considerado como uno de los municipios de mayor desarrollo económico e incluso en los últimos años ha tenido las calificaciones más altas en el Índice de Desarrollo Humano, lo cual permite constatar inicialmente que se trata de dos municipios que pueden lograr un buen nivel de desarrollo administrativo en materia de modernización. 
capacidades para absorber el cambio constante. Las facultades que tiene una sociedad para dotarse de instituciones que sean capaces de absorber el cambio, signo de nuestro tiempo, es el quid de la modernización. En ese sentido, la modernización administrativa del Estado consiste en la creación de capacidades requeridas para absorber y adaptarse a un entorno siempre cambiante. Del mismo modo, además de la ruptura con esquemas obsoletos, la modernización significa el uso, aprovechamiento y desarrollo de medios que el propio ingenio plantea como mecanismos de control y adaptación evolutiva, lo cual considera: el reconocimiento de una necesidad de cambio, adaptación, innovación y superación de las condiciones obsoletas, que en determinado momento implican un riesgo de permanencia; busca la construcción de modelos organizativos capaces de una adaptación y autorregulación en el presente, una disposición y capacidad de adecuaciones futuras, incorporando así los medios que faciliten la realización de procesos en una dinámica consistente para sus fines (Padilla y Montes de Oca, 2009:35). Dichas situaciones muestran que la modernización no es una moda o tendencia pasajera; sino una herramienta útil para que un aparato estatal sea efectivo, eficiente y eficaz, donde el ciudadano perciba el buen funcionamiento de la administración pública y sea declarante de que los servicios públicos se prestan con calidad y oportunidad.

En consecuencia con lo expuesto, en esta investigación se parte del supuesto que la modernización administrativa es una mejora del funcionamiento de la administración pública y al mismo tiempo de las capacidades gubernamentales, que tienden más hacia la eficacia y cumplimiento de las metas colectivas. Donde la modernización no es un tema ajeno a las preocupaciones de los ciudadanos, por el contrario, debe ser entendida como una respuesta a una demanda social.

Durante las últimas décadas, un sinnúmero de países han llevado a cabo programas de modernización ${ }^{3}$. Entre los factores que explican la rápida transformación de las sociedades se incluye: Los cambios tecnológicos, especialmente en las áreas de la información y las comunicaciones; el desarrollo económico, que se traduce en incrementos de los niveles de vida; el desarrollo cultural, que se transforma en mayor calidad y alcance de la educación personal; la creciente madurez po-

3 En el caso de "la historia reciente de México se ha construido por la ruptura y no por la continuidad, por lo que existe la preocupación de no tener modelos de referencia que nos ayuden a interpretar la magnitud y el sentido del cambio" (Padilla y Montes de Oca, 2009: 35). 
lítica del ciudadano, que exige cada vez mayor representatividad, transparencia, responsabilidad, en una palabra, mayor apertura democrática de las instituciones políticas (Pichardo, 2004:58). Por lo mismo, el gobierno debe implementar nuevas tecnologías administrativas, con el fin de dar respuesta a las demandas y generar mejores condiciones para alcanzar niveles de modernización competitivos en el mundo globalizado y cada vez más exigente.

Es por ello que la administración pública debe tener presente su contexto y la manera que éste se interrelaciona con los diversos sectores y recursos que conforman su ambiente. De esa manera, se podrá percatar que la sociedad está incluyendo en su actuar cotidiano a las tecnologías y la información, que son indudablemente un paso objetivo para la construcción de nuevas formas de interrelación entre los sectores que conforman el gobierno y le dan soporte, teniendo presente que el actuar gubernamental corresponde a la interacción gobierno-ciudadano en la elaboración de sus políticas públicas y en la atención de las necesidades de este último mediante la prestación de servicios públicos que le competen.

\section{REVOLUCIÓN DE LA INFORMACIÓN Y LAS TIC COMO UNA HERRAMIENTA DE MODERNIZACIÓN ADMINISTRATIVA}

El comentado concepto de modernización administrativa incluye el precepto de la flexibilización organizativa y la agilización de la relación con los ciudadanos, misma que puede sustentarse en la "revolución de la información". Este concepto difunde la idea de un cambio radical de los procesos tecnológicos hasta llegar a la noción de la "era de la información", e incluso de las formas de comunicación en el proceso histórico de la humanidad. Donde las transformaciones vienen de la mano de la multiplicación de los intercambios de información y la generación de conocimiento comunicado que se refleja en diferentes espacios de la vida humana. Las organizaciones asimilan la tecnología bien porque consideran necesaria su introducción por razones de eficiencia o bien porque sus nuevas necesidades lo exigen al estar inmersos en la revolución tecnológica e informacional ${ }^{4}$, la cual se

4 Algunos autores han relacionado esta revolución tecnológica a una transformación a gran escala de las propias formas de organización social, que afecta cuestiones muy diversas: desde las relaciones entre las empresas y los flujos de capital hasta la dinámica de las relaciones personales o de los movimientos sociales (Castells, 19964; Minc, 1987; Drucker, 1987, Welp, 2007). La 
encuentra como principal base para los nuevos procesos de globalización y medio de difusión de grandes magnitudes.

Al mismo tiempo, las marcadas diferencias en cuanto al uso de las tecnologías de la información y la comunicación (TIC) entre los países industrializados y las naciones en desarrollo han llevado a hablar de una "brecha digital". Tal concepto implica que una relativa falta de acceso a las TIC es en sí misma una desventaja estratégica que puede -y debería- abordarse de una manera distinta y separada de la miríada de problemas del desarrollo. Se considera que los males de la administración pública surgen de sus deficientes herramientas de gestión, y de una cultura organizacional que no premia la obtención de resultados ni la innovación, razón por la cual, las soluciones que se proponen están centradas, casi exclusivamente, en el terreno gerencial.

La tecnología debe situarse al servicio de la modernización de la administración pública, en lugar de diseñar la modernización en función de la tecnología. Las posibilidades de transformación de la administración pública utilizando las nuevas tecnologías son espectaculares y los gobiernos pioneros de la administración electrónica como los de Australia o Canadá, no sólo muestran con orgullo sus logros y los comparten con la comunidad internacional, sino que refuerzan constantemente sus ya de por sí ambiciosos planes de gobierno electrónico (Porrúa, 2003:2).

El Estado ha percibido la urgencia de su estructura y acción, y se propone un cambio profundo en la forma en que ciudadanos, empresas y administraciones públicas se relacionan entre sí. La idea de incorporar a las TIC como posibles alternativas es una gran innovación, ya que estas han venido desempeńado un papel relevante desde décadas atrás en este proceso, con base en sus condiciones y etapa de la evolución tecnológica, lo que indica que la adaptación o incorporación de éstas a los procesos administrativos no debería tener limitantes o rechazos a las mismas, pues se utilizan diariamente, como la informática, la telefonía fija y móvil, el fax, y más recientemente el Internet. Todas ellas han cambiado de manera permanente la forma en que trabajamos, vivimos y nos relacionamos.

teoría de la sociedad red de Manuel Castells 1999 plantea que la estructura de la nueva sociedad que reemplaza a la hegemonía de la sociedad industrial está construida en torno a redes de información. La nueva sociedad informacional se apoya, pues, en estructuras interconectadas de nodos, dinámicas y abiertas, que pueden modificarse fácilmente sin que su equilibrio interno se vea amenazado. 
En esta búsqueda de la eficacia, las administraciones han realizado importantes inversiones para incorporar la utilización de las tecnologías de la información y las comunicaciones (TIC) en su quehacer diario. Estas tecnologías han permitido en distinto grado- automatizar un elevado número de procesos y procedimientos y han simplificado considerablemente el trabajo interno desarrollado por muchos departamentos, con la correspondiente mejora de su eficiencia, cumpliendo así una parte de las expectativas que la administración electrónica había despertado (Ministerio de Economía y Hacienda, 2009:15). Organismos internacionales como es el caso de la Organización para la Cooperación y Desarrollo Económico (OCDE), hacen pública la importancia de la mejora de los servicios públicos y, en particular, la incorporación de la tecnología en la prestación de los mismos.

Trabajando así en dos grandes líneas:

- Tecnologías de la comunicación (Information and Comunicación Technologies).

- Administración y Gestión Pública (Public Governance and Manangement).

Bajo sus auspicios, se reunió en Brasil, en octubre de 2007, el Foro Global sobre Administración (Global Forum on Governance), con el lema "Modernizar la Administración: estrategias y herramientas de cambio". Las conclusiones del mismo fueron que el sector público está en el centro de la transformación de la sociedad; sus nuevas formas de gestión y de prestación del servicio a través de las modernas tecnologías están cambiando el modo de funcionar de la Administración (Ministerio de Economía y Hacienda, 2009:96). Impulsando una administración más abierta y electrónica multinivel. Por lo tanto, la tecnología sería un factor determinante de la capacidad del gobierno para llevar a cabo sus tareas. Es importante resaltar que, independientemente de cuáles sean los valores u objetivos que persiga el gobierno, nuestro supuesto es que el uso de la tecnología incrementa su eficacia. La tecnología es en este sentido moralmente neutra, puede utilizarse para prohibir el acceso a sujetos no deseados y actúa como variable dependiente e independiente ante del desempeño de las instituciones. 


\section{Administración electrónica}

Las TIC son de gran importancia por los hechos que marcan en su actuar, la manera en que han impactado en la actividad del sector público, cómo han penetrado en las administraciones públicas 5 (Bellamy y Taylor, 1998), y la forma en que han generado cambios en los últimos años.

Entonces el impacto de las TIC e Internet ${ }^{6}$ en las Administraciones Públicas nos lleva a hablar de Administraciones electrónicas teniendo en cuenta la multidisciplinariedad de la Ciencia de la Administración. Existe "la necesidad de una aproximación a la e-Administración desde la Ciencia de la Administración que no caiga en el determinismo tecnológico, sino que tenga en cuenta una visión multidisciplinar y no determinista sobre la interacción entre las tecnologías y la sociedad y, por tanto, entre las tecnologías y las Administraciones Públicas, cada vez más necesaria ante el reto que supone Internet para analizar este fenómeno" (Criado, 2003:5).

Con la incorporación de las TIC en las administraciones públicas han proliferado definiciones que buscan ponerle una etiqueta a las nuevas formas de hacer -administrar, participar, gobernar- mediante las TIC. Por ejemplo: e-gobierno ${ }^{7}(e-$ government), la e-gobernanza (e-governance), la e-democracia (e-democracy) o la eadministración son las etiquetas más difundidas. Las definiciones se superponen y apenas es posible delimitar ámbitos específicos de cada una (Welp, 2007:26). En parte esta confusión conceptual puede explicarse por el hecho de que en todos los conceptos, tal como lo señala Welp, fue el sector privado el que buscó propagar a las TIC y además es el creador de la mayor parte de estas definiciones.

5 Entre los años 1940 y 1950 aparecen los primeros ordenadores modernos orientados, sobre todo, al cálculo matemático. Estos ordenadores fueron los predecesores de los llamados mainframes. En los años 1960 estas máquinas fueron incorporadas en las Administraciones Públicas.

6 Las TIC e Internet y sus capacidades para comunicar información sin restricciones espaciales, en tiempo elegido y a bajo coste, se han convertido en catalizadores de las transformaciones en curso dentro de la Sociedad de la Información. La Sociedad de la Información se refiere a nuevas estructuras en red, en las que están también presentes las Administraciones Públicas, de manera que los nuevos modelos sociales se basan en la creciente importancia que han ido adquiriendo la información y la comunicación desde la década de los setenta del siglo XX en todos los órdenes de la vida humana (Castells 1999; Dutton, 1999.

7 Ver anexo 1 para ampliar información sobre las interpretaciones de gobierno electrónico. 
Siguiendo a Welp (2007:27), algunos autores muestran la importancia del sector público como mercado para el sector privado, otros ponen énfasis en la transparencia y la participación, además de los procesos internos. Es en este contexto donde los límites entre los constructos se vuelven difusos. Sólo por mencionar un ejemplo, el gobierno de Nueva Zelanda la ha definido como "la manera en que los gobiernos emplean las nuevas tecnologías para proporcionar a las personas un mejor acceso a la información y a los servicios gubernamentales, mejorar la calidad de los servicios y dar más oportunidades para participar en los procesos y en las instituciones democráticas" (ver Welp, 2007:26).

La Carta Iberoamericana de Gobierno Electrónico (CIGE), plantea la necesidad de cooperación entre los diferentes Estados de Iberoamérica. Por tal motivo, se expresa que "los Estados deberán prepararse para la efectiva implantación de la administración electrónica acometiendo las transformaciones organizativas que consideren necesarias, así como la progresiva implantación de sistemas, equipos y programas en las Administraciones Públicas. En tal sentido, es recomendable que los Estados: reconozcan los desarrollos propios de sistemas o sus adaptaciones como capital estatal intangible, generando mecanismos de transferencia y sistemas de apoyo, para lo cual se requiere acordar nuevos marcos regulatorios" (CIGE, 2007, punto 23, citado en Ruiz, 2009:4). Es decir, la búsqueda de hacer más eficientes y transparentes las acciones gubernamentales impulsando nuevas formas de relación entre los gobernantes y los gobernados con un enfoque en la mejora de su comunicación, otorgándoles herramientas para su mayor participación y control de la cosa pública (Ruiz, 2009:4).

También se puede definir administración electrónica como el ofrecimiento de información y la entrega de servicios gubernamentales en línea basados en Internet. Desde esta perspectiva surgen nuevas formas de operación gubernamental de las agencias públicas con los usuarios, recurriendo para ello al uso de tecnologías de información y comunicación (TIC), pero también siendo necesario considerar la relación entre gobierno y ciudadano. La extensión del concepto e-administración ${ }^{8}$ supone la necesidad de análisis con una perspectiva que supere el determinismo de algunas propuestas teóricas anteriores, teniendo en cuenta que una perspectiva

8 Por respeto a la trayectoria que trae consigo la administración pública, el concepto más utilizado para describir este tipo de fenómenos y proyectos en castellano es la administración electrónica (o e-administración). 
dentro de la Ciencia de la Administración deriva de una visión multidisciplinar de los fenómenos asociados a las Administraciones Públicas?.

Aquí, en la definición de e-administración se debe tener presente la idea que plantea Castells (1999) para apoyar al término, donde la gente, las instituciones, las empresas o las Administraciones públicas transforman la tecnología, cualquier tecnología, apropiándosela, modificándola y experimentando con ella, lo cual ocurre especialmente en el caso de la Internet, al ser ésta una tecnología de la comunicación. De este proceso de interacción surge un modelo socio-técnico, el cual va más allá de la preeminencia de las herramientas de las TIC, dándole importancia a las decisiones que se toman sobre ellas. Ya que desde este punto de partida, un análisis del uso e impacto de las TIC e Internet en las Administraciones Públicas (e-Administración) desde la Ciencia de la Administración, asumiría que las tecnologías por sí mismas no tienen las cualidades necesarias para orientar automáticamente hacia resultados concretos (ver Criado, 2003:5). Bien al contrario, organizarse y gestionar de cualquiera de las maneras que se elija es un proceso abierto para cualquier Administración Pública, dado que la existencia de una determinada tecnología, por sí sola, no determina resultados específicos (Fountain, 2001).

En suma, los planteamientos mencionados no sólo eluden el determinismo tecnológico, como ya se ha dicho, sino que tienen en mente que las Administraciones Públicas cuentan con una experiencia considerable en la gestión de tecnologías desde hace décadas y también reconocen el reto analítico que abren las redes y tecnologías más recientes; bajo este término, recurrimos a el concepto de Administración electrónica que enlaza a un todo: desde procesos técnicos, sociales, culturales, democráticos, políticos, administrativos, participación, elementos de transparencia; a todas las transformaciones que afectan al Estado-nación en el entorno de la globalización y de la emergencia de las grandes redes horizontales de comunicación interactiva.

9 El análisis de la administración electrónica se está efectuando desde un conjunto bastante heterogéneo de ámbitos disciplinarios. 


\section{LA ADMINISTRACIÓN ELECTRÓNICA Y SUS ELEMENTOS}

La Administracion Pública involucra muchos elementos para su desarrollo y no deja de lado el impulso que las tecnologías han tenido últimamente, y que estas coincidan al mismo tiempo con las últimas experiencias modernizadoras para la provisión de información, la prestación de servicios o la mejora de la atención al ciudadano, donde "los factores sociales dan forma a las tecnologías, que de la misma manera dan forma al contexto, generando una compleja retroalimentación” (Margetts, 1999, citado en Criado, 2003).

La administración electrónica ha proliferado espectacularmente dentro de las administraciones públicas y están empezando a considerar la red, tanto con respecto a sus portales públicos como en lo tocante a sus sistemas de información internos y sus intranets, como elemento clave para sus actividades y, en muchos casos, como eje vertebrador de su modernización (Aibar y Urgell, 2007:19).

Por lo tanto, la llamada Administración electrónica constituye para muchos la encarnación de esta transformación global en un terreno muy importante de la realidad social contemporánea: el Estado y sus aparatos administrativos (Aibar y Urgell, 2007:24), donde los gobiernos apoyan a las TIC para llevar a cabo sus actividades. Se calcula que en los países desarrollados, los Estados gastan entre 1 y un 1,5\% de su PIB en las TIC que utilizan los aparatos administrativos del sector público. La administración electrónica se plantea:

desde una sencilla página web, fundamentalmente informativa, que inaugura la presencia en el ciberespacio de una Administración, hasta grandes portales interadministrativos donde los usuarios pueden acceder a todo tipo de información, realizar trámites en línea, dirigirse a los representantes políticos, consultar el estado de determinados procedimientos administrativos o pagar impuestos; se extiende una gran amalgama de iniciativas, más o menos sofisticadas y con grados diversos de éxito y difusión, que muestran en la última instancia, una evidente heterogeneidad (Aibar y Urgell, 2007:25).

Las nuevas tecnologías permiten pensar en romper las reglas de funcionamiento del sector público y posibilitar una comunicación mucho más dinámica y fluida, no sólo entre las unidades de Administración, sino también entre ésta y otros actores externos, automatizando y simplificando procesos administrativos enteros, 
incorporando al sector privado y a sus formas de gestión y mejorando la eficiencia interna global de la Administración.

\section{CARACTERÍSTICAS DE LA ADMINISTRACIÓN ELECTRÓNICA}

A partir de una determinación rigurosa de los cambios y transformaciones actuales, donde las administraciones públicas hacen uso de las TIC, las características habituales de la administración electrónica son, según Aibar y Urgell (2007) que permiten la optimización y distribución de los servicios públicos, el acceso a la información pública; un gobierno orientado al ciudadano y a los resultados; la distribución de servicios públicos en línea; reducción de la burocracia; mejorar el acceso del ciudadano a la administración, y, el uso de las TIC por parte de los gobiernos y las administraciones en todas sus funciones [sin dejar a un lado la razón social de toda administración y la representación de la misma]. Aibar y Urgell (2007) se refieren a estas definiciones como aquellas que integran elementos bastante heterogéneos: desde instrumentos, el modo de emplearlos, en los objetivos o resultados, y las estrategias para conseguirlos.

Entre las ventajas que se obtienen mediante la e-administración se incluyen: la disponibilidad de interactuar con las organizaciones las 24 horas del día, facilidad de acceso sin necesidad de acudir a la oficina de manera presencial para realizar gestiones, ahorro de tiempo para realizar una gestión (Avantia, s/f). A continuación se mencionan las ventajas de la administración electrónica a partir de las consideraciones de Pichardo (2004):

- Mejorar el desempeño general de todas las actividades del gobierno; obtener mayor eficiencia en sus tareas; hacerlo más competitivo y, por tanto, contribuir a elevar la calidad de la gobernabilidad.

- La medición de resultados en la ejecución de programas y la prestación de los servicios; por tanto, lograr mayor respuesta ante la demanda ciudadana (misma que día a día se torna más exigente y compleja) con validez jurídica.

- Dar respuesta (en tiempo real) a quejas, peticiones, sugerencias y demandas ciudadanas que antes se procesaban burocráticamente, fuera de oportunidad y a un costo elevado.

- Dar participación inmediata a los ciudadanos en el combate a la mala administración, el desperdicio y la corrupción. 
- Obtener para los usuarios, los causantes o clientes, una gran variedad de servicios centrados en el ciudadano, con eficiencia, rapidez, bajo costo y comodidad, sin desplazarse de su propio domicilio.

- La posibilidad de efectuar trámites las 24 horas del día, los 365 días del año, accediendo a los mismos desde cualquier parte del país.

- Aumento de transparencia, rendición de cuentas, reducción de costos y la creación de nuevos e-servicios (Pichardo, 2004: 335-336).

Todo lo anterior conlleva a considerar las características propias del país, al igual que sus estructuras administrativas que en determinado momento condicionan la transformación institucional, aquí se plantea que el gobierno no sólo debe incorporar sistemas TIC, sino también el desarrollo de una capacidad crítica para hacer un análisis de las necesidades que existen y qué es lo que se tiene para emprender la acción (Ruiz, 2009:6; Pichardo, 2004: 335).

Finalmente, desde una perspectiva más general, Fountain (2001b) sugiere que el reto para un gobierno no estriba en implantar los servicios en línea, sino en reorganizar y reestructurar los procesos institucionales en los que se apoyan los servicios públicos. Para esta autora, internet (como nueva infraestructura de comunicación, coordinación y control) podría favorecer cambios estructurales profundos: en los procesos presupuestarios, de supervisión y en la estructura de comités y comisiones, vinculados al crecimiento de redes interdepartamentales e interadministrativas (ver Aibar y Urgell, 2007:116).

\section{La AdMinistración ELECTRÓNICA EN EL MUNDO y EN MÉXico}

La administración electrónica es una tendencia global y universal. Estados Unidos puede estar más adelantado que otras regiones, pero Europa, Asia-Pacífico y Latinoamérica están desarrollando sus propias innovaciones en materia de administración electrónica. Se constituye en una nueva manera de organizar la gestión pública para aumentar la eficiencia, transparencia, accesibilidad y capacidad de respuesta a los ciudadanos, a través del uso intensivo y estratégico de las TIC, tanto en la gestión interna del sector público como en sus relaciones diarias con ciudadanos y usuarios de los servicios públicos (Sotelo, 2006: 21). A continuación se 
presentan en el Cuadro 1 algunos ejemplos representativos de la administración electrónica en el mundo.

\section{Cuadro $\mathrm{N}^{\circ} 1$ \\ Casos representativos de Administración electrónica}

\begin{tabular}{|c|c|}
\hline Países & Alcances \\
\hline Reino Unido & $\begin{array}{l}152 \text { servicios brindados electrónicamente ( } 33 \% \text { de la totalidad de servicios. } 451 \text { servicios } \\
\text { entregados electrónicamente para el } 2005 \text {. Un millón de pequeńas y medianas empresas } \\
\text { en línea para el ańo } 2005 \text {. }\end{array}$ \\
\hline Canadá & $\begin{array}{l}4 \text { millones de solicitudes de página por semana en el sitio web de Canadá. } 100.000 \text { visi- } \\
\text { tantes diarios al Banco de Empleo. El sitio de Canadá lo consultan } 7 \text { millones por mes. }\end{array}$ \\
\hline Estados Unidos & $\begin{array}{l}\text { Al menos } 38 \text { estados y } 18 \text { municipios se encuentran como e-gobierno. Destaca el voto } \\
\text { electrónico en California. }\end{array}$ \\
\hline Nueva Zelanda & Para el 2004 se podrá acceder en línea a toda la información y servicios gubernamentales. \\
\hline España & $\begin{array}{l}\text { Un caso destacado el Departamento de Tráfico del Ayuntamiento de Barcelona que in- } \\
\text { forma sobre el tráfico y rutas de acceso adicionales. }\end{array}$ \\
\hline Singapur & $\begin{array}{l}50 \% \text { de los servicios públicos pueden ser proveídos electrónicamente, ya están en línea } \\
\text { (330 servicios). Toda interacción se podrá hacer a través del Internet en } 2005 \text {. }\end{array}$ \\
\hline Australia & $\begin{array}{l}\text { En este país se desechan anualmente } 200.000 \text { computadoras. Mediante un programa } \\
\text { gubernamental provee de equipos reciclados a comunidades de escasos recursos. }\end{array}$ \\
\hline Pakistán & $\begin{array}{l}\text { El programa del gobierno es desarrollar sitios en cada ministerio y sus } 34 \text { divisiones } \\
\text { gubernamentales. }\end{array}$ \\
\hline Perú & $\begin{array}{l}\text { Este país con el apoyo de México (compranet) implementará un sistema de compras } \\
\text { públicas en línea. }\end{array}$ \\
\hline Brasil & $\begin{array}{l}\text { El gobierno brasileńo instalará kioscos a Internet en } 4.000 \text { oficinas de correo de ciudades } \\
\text { con más de } 10.000 \text { habitantes. }\end{array}$ \\
\hline Chile & $\begin{array}{l}\text { Es uno de los países más avanzados en gobierno electrónico. Una gran mayoría del pago } \\
\text { de impuestos se realiza por medio del internet. }\end{array}$ \\
\hline
\end{tabular}

Fuente: elaboración propia.

Muchas aplicaciones innovadoras están siendo utilizadas en todo el mundo: Chile tiene un portal integrado que permite a los usuarios ingresar a las diversas entidades de su gobierno; México ofrece información turística muy variada en sus sitios, SAT, COMPRANET, SEP, SIN, entre otros; Brasil brinda a sus ciudadanos la posibilidad de hacer preguntas por vía electrónica; República Dominicana cuenta con una oficina nacional para el control del narcotráfico, con un vínculo mediante el cual los ciudadanos pueden hacer denuncias de manera anónima (West, 2009:235-236). 
En el caso de Canadá existe una tradición en lo relativo a las consultas en línea. El Plan Verde del gobierno de Mulroney o las consultas públicas sobre las medidas presupuestarias del Ministro de Finanzas, Paul Martín, son dos ejemplos de ello (Richard, 2009:215). Desde el lanzamiento de su sitio web, en 1994, el Departamento de Finanzas, publicaba informes en la Internet con el fin de llegar a mayor número de personas. A lo largo de los diez ańos siguientes, los departamentos llevaron a cabo muchas consultas en línea, con lo que recabaron gran cantidad de información. Por ejemplo, en 2002 cerca de 28 mil canadienses participaron en una encuesta en línea y examinaron diversos escenarios para el futuro de los servicios de salud. Más tarde, en 2004, la revisión de la Política sobre Comunicación del Gobierno de la Junta del Tesoro dio lugar a la creación del portal permanente llamado Consultas a los Canadienses, así como a una serie de procedimientos de deliberación y participación ciudadanas, tanto en línea como fuera de línea.

\section{Administración eleCtrónica en México}

En el gobierno de Vicente Fox, uno de los puntos de la Agenda del Buen Gobierno -un gobierno digital-impulsó la utilización óptima de las tecnologías de información y de comunicaciones para hacer más eficiente la gestión gubernamental, proporcionando servicios de mayor calidad y oportunidad a la ciudadanía, para transparentar la función pública en todos los ámbitos de gobierno y al mismo tiempo, combatir las prácticas de corrupción de la administración pública federal (Sotelo, 2009: 241).

Sin embargo, en prácticamente todas las secretarías de Estado se cuenta con una página en Internet donde se proporciona información sobre su sector y los servicios que ofrecen a la ciudadanía. De las entidades paraestatales del gobierno, 120 cuentan también con un sitio en la red, donde presentan información correspondiente a sus atribuciones y ámbitos de competencia. Y todos los gobiernos estatales brindan información a través de un sitio electrónico sobre las diferentes actividades económicas de su región, su industria, lugares turísticos más importantes, así como sobre la administración estatal. El reporte global sobre aptitud de e-gobierno 2004, dado a conocer por la ONU en el mes de diciembre de ese año, ubica a México en el sitio 30 entre 191países evaluados. Además, lo coloca 
en el décimo primer lugar en materia de sofisticación y madurez de servicios gubernamentales en línea; en sexto lugar en e-participación, y reconoce que México es una de las 10 naciones que ha progresado de manera más efectiva y rápida en su estrategia de e-gobierno (Sotelo, 2009:248).

Hasta junio de 2005 la ciudadanía tuvo acceso a 1225 trámites y servicios electrónicos gubernamentales, proporcionados por las dependencias y entidades de la administración pública federal mediante los siguientes esquemas: portales de internet, centros de atención telefónica, quioscos y ventanillas de atención al ciudadano. Una de las principales herramientas del gobierno electrónico fue el Portal Ciudadano www.gob.mx, en el que se reúnen en un solo sitio virtual los servicios y recursos informáticos más relevantes del gobierno federal. Para facilitar su consulta, presenta de manera ordenada todos los recursos informativos, servicios y trámites gubernamentales de más alto efecto, clasificándolos por temas y necesidades del ciudadano. En el periodo enero-junio de 2005 se registraron en promedio más de 20 mil visitas diarias a este portal, cifra $42,8 \%$ mayor a la observada en igual periodo del año de 2004, lo que revela la utilidad para realizar trámites y consultas a la oferta de servicios públicos (Sotelo, 2009: 244).

Otros sitios relevantes desarrollados en el gobierno de Vicente Fox fueron: el IMSS donde las empresas llevan un control sobre la afiliación de sus trabajadores; el Expediente Clínico Electrónico del IMSS, el e-SAT había recibido de mayo de 2004 a mayo de 2005, un total de 286.617 declaraciones correspondientes a personas morales, que representaban 99,8\% de los grandes contribuyentes; el portal del Infonavit recibió hasta junio de 2005 cerca de 7,5 millones de visitas; trámites electrónicos para exportadores e importadores de Bancomext; CFEmático facilita el pago de los servicios de la Comisión Federal de Electricidad; los programas de empleo y de vinculación laboral denominados Chambatel y Chambanet de la Secretaría del Trabajo y Previsión Social; a partir de octubre de 2004 el Programa de Vinculación del Portal Ciudadano del Gobierno Federal había incorporado a siete estados (Aguascalientes, Baja California, Campeche, Coahuila, Chiapas, Chihuahua y Guanajuato); y el Sistema Electrónico de Contrataciones Gubernamentales (Compranet) en los primeros seis meses de 2005, se registraron 12.574 licitaciones públicas, de las cuales 40,5\% (5.090 licitaciones) se realizaron por vía electrónica. 


\section{Estudio empírico de los municipios de Toluca y Metepec}

Los municipios se eligieron para conocer los adelantos y rezagos de la administración electrónica municipal en el Estado de México, en este caso dos gobiernos municipales significativos: Toluca y Metepec.

El primero por ser capital del Estado de México, el más poblado y con el producto industrial mayor del país. En cuanto a Metepec, se trata de un municipio de muy rápido y reciente desarrollo urbano, de tipo residencial medio y alto. En ambos casos se consideraron estos indicadores como factores de modernización administrativa dada la dinámica de cambio e innovación que se manifiesta en ambos sitios. Para complementar el análisis se llevaron a cabo dos entrevistas con los funcionarios que impulsan la tecnología e innovación administrativa de cada municipio.

El análisis que se presenta a continuación se dimensiona en un cuadro que se explica de la siguiente manera: en principio cuenta con tres columnas, siendo la primera la que se refiere a niveles de desarrollo de los portales electrónicos, misma que se divide en cuatro niveles: el primero corresponde al portal electrónico (sitio web) del municipio, el segundo se refiere a si muestra que el gobierno tiene información para la ciudadanía, en el tercer nivel se dirige a si se pueden efectuar trámites desde cualquier equipo de cómputo o en cualquier otro espacio y en el cuarto nivel se analizan las herramientas que tiene la administración electrónica para la interacción gobierno-ciudadano.

La segunda columna está prevista para describir y analizar lo referente a la modernización administrativa, mediante el análisis de los portales electrónicos de los dos gobiernos municipales de acuerdo a los niveles de desarrollo, en donde se vayan ubicando las herramientas (iconos) en cada uno de los niveles. En la tercera columna referida a los comentarios, se incluye información más detallada del análisis que se efectúa en cada nivel de desarrollo de la Administración Electrónica. De esta manera, en el cuadro 2 se presenta el análisis en donde será posible advertir la mayor o menor suficiencia y calidad de las herramientas de estos dos gobiernos locales del Estado de México. 


\section{Cuadro 2}

\section{Análisis de los logros en Administración Electrónica del Municipio de Toluca}

\begin{tabular}{|c|c|c|}
\hline $\begin{array}{l}\text { Niveles de } \\
\text { desarrollo }\end{array}$ & $\begin{array}{c}\text { Modernización administrativa } \\
\text { mediante el análisis de la } \\
\text { página electrónica }\end{array}$ & Comentarios \\
\hline $\begin{array}{l}\text { Portal electrónico del } \\
\text { municipio (sitio web) }\end{array}$ & $\begin{array}{l}\text { El municipio cuenta con su página electró- } \\
\text { nica que ha sido modificada con la llegada } \\
\text { del nuevo gobierno. Dispone devariosico- } \\
\text { nos: ayuntamiento, comunicación, trans- } \\
\text { parencia, municipio, trámites y servicios, } \\
\text { organismos y contacto. } \\
\text { En forma destacada tiene links } \\
\text { con pago de predial, geolocaliza- } \\
\text { dor, quejas, derechos humanos y } \\
\text { boletín empresarial. }\end{array}$ & $\begin{array}{l}\text { Todos estos iconos y links, muestran que } \\
\text { el gobierno de Toluca ha impulsado in- } \\
\text { formación diversa para tratar de comu- } \\
\text { nicarse con la sociedad, sin embargo, la } \\
\text { gran mayoría de esta información corre } \\
\text { del gobierno al ciudadano y en muy po- } \\
\text { cos casos, del ciudadano al gobierno. }\end{array}$ \\
\hline $\begin{array}{l}\text { Información del go- } \\
\text { bierno a la ciudadanía }\end{array}$ & $\begin{array}{l}\text { Básicamente los iconos y links están } \\
\text { diseńados para proporcionar informa- } \\
\text { ción a la ciudadanía y algunos mues- } \\
\text { tran que la sociedad se comunica con } \\
\text { el gobierno, aunque el número es } \\
\text { todavía reducido. }\end{array}$ & $\begin{array}{l}\text { En este nivel de desarrollo se puede se- } \\
\text { nalar que es adecuado, pues la mayoría } \\
\text { de sus iconos y links son de información, } \\
\text { aunque pudieran ser en un mayor núme- } \\
\text { ro y que ello permita que la ciudadanía } \\
\text { esté mejor informada en todos los rubros } \\
\text { que le son necesarios y convenientes. }\end{array}$ \\
\hline $\begin{array}{l}\text { Trámites y } \\
\text { servicios }\end{array}$ & $\begin{array}{l}\text { Existe una cantidad importante de trá- } \\
\text { mites señalados en la página electrónica, } \\
\text { bastaría ver las áreas y unidades admi- } \\
\text { nistrativas, espacio donde se muestran } \\
\text { los trámites de cada una de ellas y que } \\
\text { son enunciativos, lo que quiere decir } \\
\text { que tienen un esquema eminentemente } \\
\text { tradicional. Los trámites que pueden rea- } \\
\text { lizarse por la vía electrónica son el pago } \\
\text { del predial y el pago de agua, para otros } \\
\text { trámites el ciudadano tendrá que acudir } \\
\text { en forma tradicional a realizarlo. }\end{array}$ & $\begin{array}{l}\text { En virtud de que sólo se pueden hacer } \\
\text { dos trámites por la vía electrónica desde } \\
\text { el inicio hasta el final (efectuar el pago y } \\
\text { que se emita el comprobante), se percibe } \\
\text { la situación de que el nivel de desarrollo } \\
\text { en este aspecto y tomando como base el } \\
\text { referente de la modernización, es todavía } \\
\text { inicial. }\end{array}$ \\
\hline $\begin{array}{l}\text { Interacción } \\
\text { gobierno / } \\
\text { ciudadano }\end{array}$ & $\begin{array}{l}\text { Existen dos mecanismos para que el } \\
\text { ciudadano pueda acceder a informa- } \\
\text { ción gubernamental y el gobierno } \\
\text { proporcione la respuesta respectiva. } \\
\text { No obstante, la información no es la } \\
\text { suficiente, además existe otra que se } \\
\text { considera legalmente confidencial. } \\
\text { Esos mecanismos son: IFOEM vía la } \\
\text { obligación de transparentar los gobier- } \\
\text { nos, y la del SICOSIEM, que es la so- } \\
\text { licitud para institucionalmente consultar } \\
\text { y pedir la información que se requiere } \\
\text { del gobierno municipal. Se puede estar } \\
\text { de acuerdo que en términos de moderni- } \\
\text { zación existe un avance, en donde el ciu- } \\
\text { dadano sepa lo que su gobierno realiza. }\end{array}$ & $\begin{array}{l}\text { La interacción es todavía muy limitada, } \\
\text { ya que son a través de mecanismos obliga- } \\
\text { torios vía las solicitudes de transparencia, } \\
\text { siendo necesario disponer de otras herra- } \\
\text { mientas para fortalecer la interacción con } \\
\text { el ciudadano. }\end{array}$ \\
\hline
\end{tabular}

Fuente: elaboración propia con información de la página electrónica del municipio de Toluca. Consulta primera quincena de junio del 2010. www.toluca.gob.mx 
Como se puede apreciar, en el caso de Toluca, en la información que se proporciona a la ciudadanía todavía están pendientes herramientas que permitan al gobierno intensificar el trabajo del equipo de los profesionales de la Administración Pública con los expertos del área técnica, a efecto de diseñar y poner en operación todos los procesos del portal a fin de que el ciudadano se informe y adicionalmente pueda realizar trámites desde su computadora. En la capital del Estado, la implementación del programa "Toluca en línea", busca disminuir la incomunicación entre el gobierno y los ciudadanos, "implementando redes sociales que permitan la flexibilidad de horario, teletrabajo e interacción ciudadana."

La Administración Electrónica requiere una inversión, siendo a la vez un ahorro para el ciudadano, quien no tendrá necesidad de desplazarse a las oficinas receptoras de los impuestos. Respecto a los servicios que se prestan vía administración electrónica, la mayoría son de información y trámites, pero como e-servicios en el municipio de Toluca destacan el pago de predial y recientemente el pago del agua como un e-servicio completo. Por lo tanto, para estos pagos, el ciudadano tiene la opción de no hacerlos en oficinas físicamente. Se pretende, en un futuro próximo, ampliar la gama con la atención ciudadana personalizada, como servicio de alertas viales, interacción con los servidores públicos, traslado de dominio, bolsa de trabajo, pago de solicitudes de uso de suelo y licencia de construcción. ${ }^{10}$ Asimismo, implementar foros a través de blogs, chats y brindar a la ciudadanía la posibilidad de reportar en línea fallas de luz, baches, etc.

La administración de Toluca espera que los ciudadanos realicen sus pagos en forma electrónica en un $20 \%$, con un crecimiento anual del $15 \%$. Conforme al pago de predial vía electrónica se busca alcanzar una recaudación del $10 \%$ anual. También se reconoce que falta cultura informática por parte de los ciudadanos, dado que prefieren desplazarse a las oficinas para recoger su recibo oficial, por desconfianza no utilizan la administración electrónica para hacer el pago. Otro de los obstáculos es que no todos los ciudadanos cuentan con tarjeta de crédito o débito, por tanto se siguen apegando a la costumbre y al método tradicional. Según se informó, el portal de Toluca cuenta con 250 visitas diarias y siguen una proyección en aumento, gracias a la incorporación de mecanismos de interacción ciudadana. Adicionalmente, se ha puesto en práctica el servicio de módulos multipago ubica-

10 Información obtenida en entrevista realizada el 13 de agosto de 2010 al Ing. Pedro Pallares, Subdirector de Tecnologías de la Información del H. Ayuntamiento de Toluca. 
dos en zonas estratégicas de la ciudad, con lo que se busca tener una alternativa más para facilitar a los ciudadanos la realización de pagos. Finalmente, se destaca que aún falta mucho por hacer ya que frecuentemente los portales electrónicos quedan en el olvido, o no existe una política de actualización constante, lo que reduce su utilidad.

\section{Municipio de Metepec: AnÁlisis de los logros de la Administración ELECTRÓNICA}

En el caso de Metepec, se presenta un nivel de e-servicios más desarrollado, sobre todo en la información que se le brinda a la población, puesto que tiene en su portal electrónico información que es apta para ser consultada por el ciudadano, enseguida se presenta el cuadro de análisis de su página web.

El municipio de Metepec, cuenta con dos e-servicios que corresponden al pago de predial y agua; es necesario señalar que aún hace falta incorporar a esta dinámica una mayor cantidad de servicios, ya que la mayoría continúa prestándose bajo esquemas tradicionales.

En lo que se refiere a la interacción, este gobierno tiene un mejor desarrollo que el de Toluca, puesto que cuenta con mayor número de herramientas (iconos) mediante los cuales el ciudadano puede interactuar con su gobierno y viceversa. Punto que merece reconocimiento es que en este renglón, la interacción puede darse mediante las redes sociales twitter, facebook y hi5, es posible promover que los ciudadanos interactúen entre sí, pero también es posible que el gobierno participe en estas redes sociales y se pueda conocer las opiniones de los ciudadanos con relación a lo que hace, o lo que le falta por hacer. Esto lo coloca como un gobierno abierto, transparente y que propende a un mejor nivel de democracia y legitimidad. 


\section{Cuadro 3 \\ Análisis de los logros de la Administración Electrónica del Municipio de Metepec}

\begin{tabular}{|c|c|c|}
\hline $\begin{array}{l}\text { Niveles de } \\
\text { desarrollo }\end{array}$ & $\begin{array}{c}\text { Modernización administrativa } \\
\text { mediante el análisis de la } \\
\text { página electrónica }\end{array}$ & $\begin{array}{l}\text { Comentarios finales y } \\
\text { asignación de calificación }\end{array}$ \\
\hline $\begin{array}{l}\text { Portal electrónico } \\
\text { del municipio (sitio } \\
\text { web) }\end{array}$ & $\begin{array}{l}\text { El municipio cuenta con su página elec- } \\
\text { trónica que ha sufrido modificaciones a } \\
\text { la llegada del nuevo gobierno municipal. } \\
\text { Dispone de varios iconos como son: pre- } \\
\text { sidencia, dependencias, trámites, turismo, } \\
\text { eventos, transparencia y Metepec en vivo. } \\
\text { Destacan varios links: pago de agua, } \\
\text { pago de predial, oficina virtual de em- } \\
\text { pleo, transparencia, innovación guber- } \\
\text { namental, boletines electrónicos, vengan } \\
\text { esos cinco, censo de población, agen- } \\
\text { da, sala de prensa, galería, multimedia, } \\
\text { compromisos, cultura, premio ox cali- } \\
\text { dad web, Sicosiem, Sat, así como varios } \\
\text { enlaces más. }\end{array}$ & $\begin{array}{l}\text { Todos estos iconos y links muestran que } \\
\text { el gobierno de Metepec ha impulsado } \\
\text { información de diversa índole para tra- } \\
\text { tar de comunicarse con la sociedad, sin } \\
\text { embargo, la gran mayoría de esta infor- } \\
\text { mación se efectúa del gobierno al ciuda- } \\
\text { dano. }\end{array}$ \\
\hline $\begin{array}{l}\text { Información del } \\
\text { gobierno a la ciu- } \\
\text { dadanía }\end{array}$ & $\begin{array}{l}\text { Básicamente los iconos y links están di- } \\
\text { seńados para proporcionar información a } \\
\text { la ciudadanía, y algunos muestran que la } \\
\text { sociedad si requiere alguna información, } \\
\text { o efectuar algún trámite, para ello debe } \\
\text { comunicarse con el gobierno, puesto que } \\
\text { algunos iconos permiten o no que se efec- } \\
\text { túe todo el procedimiento de algún trá- } \\
\text { mite, y de esta manera continuar con una } \\
\text { administración tradicional. }\end{array}$ & $\begin{array}{l}\text { Este nivel de desarrollo se puede seńalar } \\
\text { que es adecuado, pues la mayoría de sus } \\
\text { iconos y links son de información, aun- } \\
\text { que pudieran ser en un mayor número } \\
\text { para que la ciudadanía esté mejor infor- } \\
\text { mada. }\end{array}$ \\
\hline $\begin{array}{l}\text { Trámites y } \\
\text { servicios }\end{array}$ & $\begin{array}{l}\text { Existe una cantidad importante de trámi- } \\
\text { tes seńalados en la página electrónica, bas- } \\
\text { taría ver las áreas administrativas dentro } \\
\text { del link de transparencia, espacio donde } \\
\text { se muestran los trámites de cada una de } \\
\text { ellas y que son enunciativos, lo que quiere } \\
\text { decir que funciona a la usanza tradicional. } \\
\text { Los únicos trámites posibles por la vía } \\
\text { electrónica son el pago del agua y del } \\
\text { predial, donde se manifiesta en primera } \\
\text { instancia la interacción del gobierno al } \\
\text { ciudadano y posteriormente, del ciuda- } \\
\text { dano al gobierno. No obstante y pese a } \\
\text { dicha facilidad, la página debería tener un } \\
\text { mayor número de trámites que se pudie- } \\
\text { ran realizar y no sólo colocar la informa- } \\
\text { ción de qué documentos y qué requisitos } \\
\text { son necesarios para realizar algún trámite, } \\
\text { donde el ciudadano tenga que ocurrir a } \\
\text { efectuar el trámite a la oficina respectiva. }\end{array}$ & $\begin{array}{l}\text { En virtud de que solo se pueden hacer } \\
\text { dos trámites por la vía electrónica desde } \\
\text { el inicio hasta el final (efectuar el pago } \\
\text { y que se emita el comprobante), plantea } \\
\text { la situación de que el nivel de desarrollo } \\
\text { en este aspecto y tomando como base el } \\
\text { referente de la modernización, es todavía } \\
\text { inicial, puesto que carece de otros trámi- } \\
\text { tes que se efectúan de la misma forma, es } \\
\text { decir tradicionalmente. } \\
\text { Asimismo, es necesario que los trá- } \\
\text { mites por la vía electrónica sean } \\
\text { puestos a prueba antes de colocar } \\
\text { su servicio en la página, ya que pue- } \\
\text { den presentar dificultad al no con- } \\
\text { cretar todo el proceso del trámi- } \\
\text { te, y solo quedarse en la obtención } \\
\text { del deducible a pagar. }\end{array}$ \\
\hline
\end{tabular}




\begin{tabular}{|c|c|c|}
\hline $\begin{array}{l}\text { Niveles de } \\
\text { desarrollo }\end{array}$ & $\begin{array}{l}\text { Modernización administrativa } \\
\text { mediante el análisis de la } \\
\text { página electrónica }\end{array}$ & $\begin{array}{l}\text { Comentarios finales y } \\
\text { asignación de calificación }\end{array}$ \\
\hline $\begin{array}{l}\text { Interacción ciuda- } \\
\text { dano-gobierno }\end{array}$ & $\begin{array}{l}\text { Existen dos mecanismos los cuales, en } \\
\text { términos de normatividad, funcionan } \\
\text { para que el ciudadano pueda acceder a } \\
\text { información gubernamental y el gobierno } \\
\text { proporcione la respuesta respectiva. } \\
\text { No obstante, la información no es la su- } \\
\text { ficiente, además existe otra que se consi- } \\
\text { dera legalmente confidencial. Esos me- } \\
\text { canismos son Ifoem vía la obligación de } \\
\text { transparentar los gobiernos, y la del Sico- } \\
\text { siem que es la solicitud para institucional- } \\
\text { mente consultar y pedir la información } \\
\text { que se requiere del gobierno municipal. } \\
\text { Existe un buen avance en términos de } \\
\text { modernización para que el ciudadano } \\
\text { sepa lo que su gobierno realiza. También } \\
\text { existen las redes sociales como son: hi5, } \\
\text { twitter y facebook que permiten la inte- } \\
\text { racción entre ciudadanos o al menos sir- } \\
\text { ve para que la autoridad puede saber lo } \\
\text { que opinan éstos. } \\
\text { La página contiene iconos y links que } \\
\text { permiten acceder a la información guber- } \\
\text { namental de dos formas; la primera, es la } \\
\text { interacción del gobierno al ciudadano, y } \\
\text { algunos de los iconos son: transparencia } \\
\text { de trámites y servicios (descripción del } \\
\text { trámite, requisitos, pasos a seguir, horario } \\
\text { de atención, costo, tiempo de respuesta, } \\
\text { documento a recibir), agenda o itinerario } \\
\text { de eventos, boletines electrónicos, capa- } \\
\text { citación y asesoría para obtener empleo. } \\
\text { Por otra parte, se aprecia interacción del } \\
\text { ciudadano al gobierno, mediante algunos } \\
\text { de sus iconos como son: Sicosiem, chat en } \\
\text { línea, web mail, quejasydenuncias, twitter, } \\
\text { facebook, hi5. }\end{array}$ & $\begin{array}{l}\text { Estos dos mecanismos son los que per- } \\
\text { miten que el ciudadano se comunique y } \\
\text { consulte información del gobierno mu- } \\
\text { nicipal, pues bajo este mecanismo son } \\
\text { los que obligan al gobierno a transparen- } \\
\text { tar sus acciones, procesos, estructuras, } \\
\text { normatividad, programas, planes y re- } \\
\text { sultados, sin embargo, se apegan a la re- } \\
\text { glamentación establecida rigurosamente, } \\
\text { sin que existan posibilidades de una ma- } \\
\text { yor fluidez en la información, es decir, del } \\
\text { gobierno al ciudadano. } \\
\text { No se observan otros esquemas y meca- } \\
\text { nismos de interacción gobierno - ciuda- } \\
\text { danía y ciudadanía - gobierno para con- } \\
\text { formar un ambiente de comunicación } \\
\text { permanente. }\end{array}$ \\
\hline
\end{tabular}

Fuente: elaboración propia con información de la página electrónica del municipio de Metepec. Consulta primera quincena de junio del 2010. www.metepec.gob.mx. 
En este gobierno municipal, el impulso que se está dando a la Administración Electrónica, va referido principalmente a la implementación del uso de las TIC e Internet al interior de la administración municipal como un flujo de información entre sus dependencias, y para estar a la vanguardia se realizó una importante inversión en infraestructura de comunicación y equipo, lo que a la larga generará ahorros de tiempo, esfuerzo y oportunidad de información. Conforme a los servicios que se prestan por Administración Electrónica, se reconoce que sólo cuentan con servicios de información en cuanto a trámites y procedimientos, para esta administración es relevante la interacción ciudadano-gobierno (solicitudes, denuncias, transparencia, etc.).

Un servicio que se puede realizar por completo en el portal es el pago de predial, en un 5\% de la recaudación registrada en el año, expresando que este porcentaje se incremente anualmente. También se cuenta con el servicio de pago de agua vía Internet, nuevo en esta administración. La recaudación de estos dos impuestos vía electrónica permitirá el aumento de esta en un 10\%, impulsando campañas de regularización de pago, según se informó en los meses de febrero a junio de 2010, se tuvieron 48.000 consultas lo que la hace ver como una herramienta útil y necesaria para los ciudadanos. Se indicó, que se están revisando los procesos administrativos, para poder implementar reingeniería de estos y ofrecerlos a los ciudadanos de una manera más ágil como servicios vía electrónica ${ }^{11}$. Finalmente, en los e-servicios el pago de predial y agua se monitorean para corregir los errores que se van presentando, donde también los comentarios de los ciudadanos son instrumentos de mejora de la Administración Electrónica.

En virtud de lo anterior, el análisis derivado de esta clasificación de niveles de desarrollo de los portales electrónicos de los municipios, mismos que se traducen en políticas de modernización administrativa, específicamente en el terreno de la administración (pública) electrónica, permite percibir que ambos gobiernos, al inicio de esta administración municipal (2009-2012) ${ }^{12}$, coinciden en que el esfuerzo en esta materia de gobierno es importante. Se observa la necesidad de evaluar a sus gobiernos, de convencer a los servidores públicos y a los usuarios de la importancia de la utilización de la Administración Electrónica, puesto que el

11 Información obtenida en entrevista realizada en agosto de 2010 al Lic. Ramón Cuevas, Director de Innovación gubernamental del H. Ayuntamiento de Metepec.

12 Dichos gobiernos cumplen un año de gestión en agosto de 2010. 
propósito es lograr varias metas; entre las más importantes: la transparencia de los procesos, políticas, acciones y metas alcanzadas, reducir el nivel de corrupción, cancelar el intermediarismo en los trámites administrativos, modificar la cultura del servidor público y de la ciudadanía en la utilización de la administración electrónica en todas sus expresiones en información, gestión y diálogo permanente que fortalezca la gobernabilidad y la democracia. Por lo tanto, es pertinente decir que la interacción entre gobiernos municipales y su población es todavía muy restringida, el trecho es todavía muy largo, puesto que existen muy pocas opciones de interacción. De esta forma el ciudadano podrá evaluar los logros y carencias de la administración, y sancionarla según los beneficios que le reporta.

De manera general, se recomienda para estos dos gobiernos: seguridad de la información y de las transacciones que se realicen en cada trámite, amplitud de la información para que los ciudadanos cuenten con los datos necesarios y suficientes, acceso desde cualquier lugar a cualquier hora del día durante los siete días de la semana y medir, auditar y evaluar el programa de gobierno electrónico para hacerlo cada vez más un instrumento de eficiencia, democracia, transparencia y legitimidad.

\section{Conclusiones}

La Administración Pública debe considerar su contexto y la manera en que éste se interrelaciona con los diversos sectores y recursos que conforman su ambiente; al propiciar la interacción gobierno-ciudadano en la elaboración de sus políticas públicas y en la detección de las necesidades que el ciudadano quiere resolver mediante la prestación de los servicios públicos. El empleo de las TIC en la administración pública no deja de crecer, convirtiéndose en un elemento de mayor importancia para la modernización administrativa, por lo tanto, la administración electrónica está concebida para la obtención de la información ofrecida por los poderes públicos, para realizar trámites de los ciudadanos, reduciendo el tiempo de su gestión; también refuerza la cooperación entre las autoridades nacionales, regionales y locales. La Administración Electrónica ha surgido para permitir: optimizar la prestación de los servicios y el acceso a la información; economías de escala y que el costo unitario de cada operación del gobierno sea más bajo, mejorando la capacidad de respuesta a los ciudadanos, entre otros beneficios. 
Del análisis de los casos de estudio se deriva que en el terreno de los e-servicios de la Administración Electrónica, coinciden en que el esfuerzo en esta materia es importante. En general, se pudo conocer que Toluca considera a la implementación de la Administración Electrónica como inversión y beneficio total para la sociedad, ya que este ahorra no solo tiempo, sino gastos de traslado, aunque son muy pocos los servicios que se proporcionan en línea, donde la mayoría se centran en servicios de información y divulgación de actividades. La administración está considerando impulsar campañas para que la ciudadanía utilice los medios electrónicos y vea sus beneficios.

En cuanto a Metepec, la administración electrónica se ve como una gran inversión monetaria, pero los beneficios los comparte tanto la administración como la población, la primera por la eficiencia y eficacia al interior de la administración que presta el servicio, donde se generan canales más rápidos de interacción y comunicación entre las diferentes dependencias, y por el otro lado la calidad del servicio hacia el ciudadano, por lo tanto, se reconoce el impulso a la administración electrónica por parte de este municipio. Sin embargo, se puede ver que el camino por recorrer es todavía largo y complejo, no sólo por la complejidad de los programas y sistemas a instrumentar en el futuro, sino desde la óptica de la consolidación de la administración electrónica en tanto logros en la utilización de la ciudadanía de los e-servicios, e-información, así como para la evaluación de los gobiernos. Por lo que hay que trabajar más en esta forma novedosa de concebir al gobierno y a la administración pública, de tal manera que los ciudadanos vean a los servicios públicos dentro de la administración electrónica como una herramienta trascendental en un futuro.

\section{REFERENCIAS BibLIOGRÁFICAS}

Aibar, Eduard y Urgell, Ferran (2007). Estado, Burocracia y Red: Administración electrónica y cambio organizativo. España: UOC, Generalitat de Catalunya.

Avantia Ingeniería de Vanguardia (s/f). E-Administración. Disponible: http://www.avantiavanguardia.com/proyectos/e_administracion.php.

Bellamy, Christine, y John A. Taylor (1998). Governing in the Information Age. Buckingham: Open University Press. 
Castells, Manuel (1999). La era de la información. Madrid: Alianza.

Criado Grande, J. Ignacio (2003). “Mitos y Realidades sobre las Tecnologías de la Información y la Comunicación e Internet en las Administraciones Públicas". Ponencia presentada en el VI Congreso de Ciencia Politica y de la Administración, Barcelona, 18-20 septiembre. Departamento de Ciencia Política y de la Administración II, Universidad Complutense de Madrid, España.

Drucker, Peter (1987). Las fronteras de la administración: donde las decisiones del mañana cobran forma hoy. Buenos Aires: Editorial Sudamericana.

Dutton, William (1999). Society on the Line. Inglaterra: Oxford University Press.

Eisenstadt, S.N. (1997). Modernización: movimientos de protesta y cambio social. Buenos Aires: Amorrortu Editores.

Fountain, Jane (2001a). Building the virtual state. Information Technology and Institutional change. Estados Unidos: The Brookings Institutions.

Fountain, Jane (2001b). "Toward a Theory of Federal Bureaucracy in the 21st Century". En Laine Ciulla Kamarck, Joseph S., Jr. Nye (editores). Governance. Com: Democracy in the Information Age. Washington, D.C.: Brookings Institution Press.

López Dávila, Irak (2002). “e-Gobierno: ¿Revolución o Moda?” Prospectiva Año 7. No 20.

Minc, Alan. (1987). "The informatization of society". En Cawkell, A. (editor) Evolution of an Information Society. Londres: Aslib.

Ministerio de Hacienda y Economía (2009). Administración electrónica y el servicio a los ciudadanos, El Ministerio de Economía y Hacienda ante los Retos de la Ley 11/2007, de Acceso Electrónico de los Ciudadanos a los Servicios Públicos. España: Ministerio de Economía y Hacienda, Real Casa de la Moneda.

Padilla, Roberto y Montes de Oca M., Juan (2009). Modernización de la Administración Pública en el Estado de México. México: Biblioteca del Bicentenario, Secretaría Técnica del Gabinete.

Pardo, María del Carmen (1992). La modernización administrativa en México. México: INAP-Colegio de México, Centro de Estudios Internacionales.

Pichardo, Ignacio (2004). Modernización administrativa: propuesta para una reforma inaplazable. México: Colegio Mexiquense.

Porrúa, Miguel A. (2003). “Elementos para la creación de una estrategia de gobierno electrónico”, ponencia presentada en VIII Congreso Internacional del CLAD sobre la Reforma del Estado y de la Administración Pública, Panamá. 
Richard, Elisabeth (2009). "Deliberación en línea en el gobierno de Canadá: organizando la 'trastienda'”. En, María del Carmen Pardo y Ernesto Velasco Sánchez (coord.). La gerencia pública en América del Norte. Tendencias actuales de la reforma administrativa en Canadá, Estados Unidos y México. México: Colegio de México, Instituto de Administración Pública de Nuevo León.

Ruiz Alanís, Leobardo (2009). "Gobierno Electrónico, un desafío para la burocracia gubernamental”, ponencia presentada en el XIV Congreso Internacional del CLAD sobre la Reforma del Estado y de la Administración Pública, Salvador de Bahía, Brasil.

Sánchez González, José Juan (2002). “Mitos y realidades del gobierno digital”. En Revista IAPEM. No. 52, pp. 69-86.

Sotelo, Abraham (2006). México: un gobierno digital en expansión. México: DPImagen.

Sotelo, Abraham (2009). “Tecnología para acercar el gobierno a los ciudadanos: la experiencia mexicana”. En, María del Carmen Pardo y Ernesto Velasco Sánchez (coord.). La gerencia pública en América del Norte. Tendencias actuales de la reforma administrativa en Canadá, Estados Unidos y México. México: Colegio de México, Instituto de Administración Pública de Nuevo León.

Welp, Yanina (2007). ¿Hacia el fin de la administración pública burocrática?: Los efectos de las TIC sobre el cambio organizativo, los casos de Cataluña, Escocia, Emilia-Romaña y Quebec. España: Universidad Pompeu Fabra.

West, Darrell (2009). “Gobierno Electrónico: ¿qué promete? ¿Qué ha conseguido?”. En, María del Carmen Pardo y Ernesto Velasco Sánchez (coord.). La gerencia pública en América del Norte. Tendencias actuales de la reforma administrativa en Canadá, Estados Unidos y México. México: Colegio de México, Instituto de Administración Pública de Nuevo León. 


\section{Anexo I \\ INTERPRETACIONES DEL GOBIERNO ELECTRÓNICO}

\begin{tabular}{c|l} 
Escuelas & \multicolumn{1}{c}{ Descripción } \\
Primera & $\begin{array}{l}\text { Derivada del enfoque de e-commerce, se explica el gobierno electrónico como la } \\
\text { transformación en la entrega de servicios gubernamentales al ciudadano a través del } \\
\text { uso de la tecnología. Esta visión y su limitado enfoque en la entrega de servicios pú- } \\
\text { blicos por la vía electrónica, es la más simplista, pero la más popular y generalizada }\end{array}$ \\
\hline Segunda & $\begin{array}{l}\text { Concibe al gobierno electrónico como el aprovechamiento de las TIC para hacer } \\
\text { más eficientes todas las áreas del gobierno. Esta escuela lo define como un "go- } \\
\text { bierno inteligente", que utiliza la tecnología de Internet para conectar las partes } \\
\text { internas del gobierno que le permitan una mejor operación integral hacia adentro } \\
\text { yhacia afuera del aparato gubernamental }\end{array}$ \\
\hline Tercera & $\begin{array}{l}\text { Considera al gobierno electrónico mucho más que una simple entrega de servi- } \\
\text { cios gubernamentales de manera electrónica y el uso de las TIC para transformar } \\
\text { las instituciones públicas. Se le concibe como una auténtica revolución ciudada- } \\
\text { na que transformará la naturaleza misma del gobierno. Se define como un nuevo } \\
\text { modelo de gobierno y de gobernanza, acorde a la sociedad de la información y el } \\
\text { conocimiento. Se propone como la revolución del siglo XXI, bajo un "Gobierno } \\
\text { Ciudadano". }\end{array}$ \\
\hline
\end{tabular}

Fuente: elaboración a partir de (López, 2002: 57).

Recibido: 20 marzo 2011 / Aprobado: 15 junio 2012 